\title{
Reverse Schottky gate current in AIGaN-GaN high-electron-mobility-transistors
}

\author{
Rik Jos ${ }^{\text {a) }}$ \\ Microwave Electronics Laboratory, Chalmers University of Technology, Kemivägen 9, 41296 Gothenburg, \\ Sweden and NXP Semiconductors, Gerstweg 2, 6534AE Nijmegen, The Netherlands
}

(Received 7 August 2012; accepted 12 October 2012; published online 8 November 2012)

\begin{abstract}
Two theoretical one-dimensional models are developed for reverse currents through Schottky gate contacts on AlGaN-GaN high-electron-mobility-transistors (HEMTs) and compared with measurement data. One model covers ideal triangular and square junction barriers and contains current contributions of thermionic emission, thermionic field emission, and tunneling of electrons from the contact metal towards the two dimensional electron gas (2-DEG) at the AlGaN-GaN interface. The second model describes the contribution of trap assisted tunneling through a Schottky barrier. Both models are compared with measurements done on AlGaN-GaN diodes at reverse voltages between threshold voltage and zero volt, which is the regime in which the current flow can be described by one-dimensional models. The trap assisted tunneling model cannot explain the data. The first model agrees with the measurements only if it is assumed that the barrier is triangular and that the current only flows through a fraction $\left(2 \times 10^{-4}\right)$ of the junction area, probably through defect patches. The triangular barrier in the defects has a barrier height of $0.58 \mathrm{eV}$. This result is consistent with previously reported findings of defect patches at the AlGaN surface. (C) 2012 American Institute of Physics. [http://dx.doi.org/10.1063/1.4764866]
\end{abstract}

\section{INTRODUCTION}

Several models have been proposed to explain reverse leakage currents in Schottky gates on AlGaN-GaN high-electron-mobility-transistor (HEMT) structures. Essentially, two different groups can be distinguished: direct current models that are based on a combination of tunneling, thermionic field emission (TFE), and thermionic emission (TE) ${ }^{1}$ and models that next to the direct current also include a current contribution from trap assisted tunneling (TAT). ${ }^{2}$ All models depend strongly on the Schottky barrier shape, i.e., the energy of the conduction band in the $\mathrm{AlGaN}$ as function of depth beneath the metal-AlGaN interface.

Direct and TAT current models have been published before in AlGaN-GaN Schottky structures ${ }^{1,3}$ in MOS structures ${ }^{4}$ and capacitors. ${ }^{5}$ There are, however, several problems with the published models that justify a new approach. The direct current models are conflicting with each other in the electron energy that is used in calculating the probability of transmission through the barrier. Some authors only take the kinetic energy directed transversal to the Schottky interface, ${ }^{6}$ while others take the total electron energy. ${ }^{1} \mathrm{We}$ will show that both approaches are incorrect and that an energy somewhere in between these values has to be used, depending on the ratio of the effective masses of the electron in the Schottky metal and in the AlGaN. In many TAT models, fitting parameters are used in the expressions for the trapping and de-trapping rates. ${ }^{3,5,7}$ These models only consider the TAT current component. In this paper, however, we are treating both the direct current and the TAT current in the same Schottky junction. Therefore, we need a unified theory

${ }^{\text {a)} E m a i l: ~ r i k . j o s @ n x p . c o m . ~}$ that treats direct and TAT currents on equal footing deriving both from the same first principles.

In this paper, we study a model for the direct current based on tunneling, TFE, and TE reverse leakage through triangular and square barriers in Sec. II. In Sec. III, we derive a model for the TAT current in a triangular barrier. As will be shown in Sec. IV, none of these models agree well with experiments if it is assumed that the total Schottky surface area contributes to the current. Some authors have suggested that the current is concentrated in numerous very small defect patches, sometimes associated with screw dislocations in the AlGaN, that have a low barrier height and carry almost all current. ${ }^{8-11}$ The rest of the surface area would have a high barrier height such that its contribution to the current can be ignored. We can use the ideal barrier model to calculate the current in these patches if we assume that there is an effective total patch area and an effective barrier height. This patch model therefore introduces one extra parameter: the effective surface area. Since we do not know how the potential and the electric field are shaped inside these patches, we have to consider various possibilities and compare the results with the measurements. In Sec. II, we describe a few possible field distributions in the patches.

The calculations of the equations that are derived in the theoretical models are performed numerically (MATHCAD).

\section{DIRECT REVERSE CURRENT THROUGH TRIANGULAR AND SQUARE SCHOTTKY BARRIERS}

\section{A. Triangular barrier}

Fig. 1 shows the triangular Schottky barrier between gate metal and AlGaN-GaN 2-DEG in case of a negative applied voltage $V_{r}$. The gate current due to tunneling and 


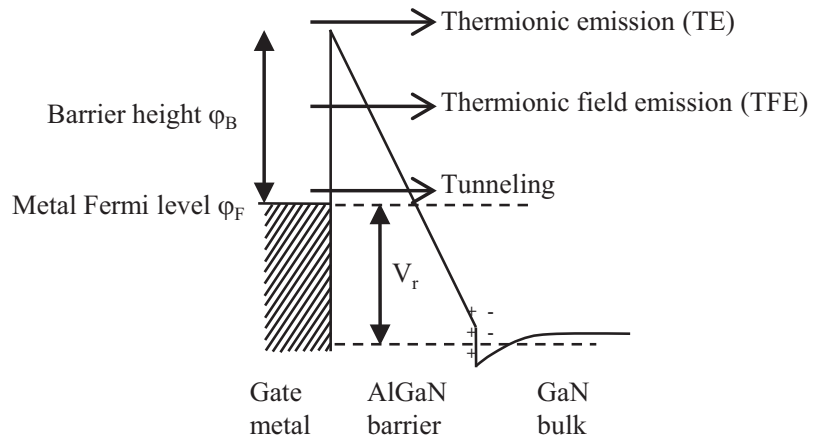

FIG. 1. Leakage current mechanisms for triangular Schottky barrier under reverse bias. Reverse bias voltage is $V_{r}$. The three current contributions, tunneling, TFE, and TE are indicated. The main contribution by tunneling takes place at the metal Fermi level.

thermionic field emission can be derived using the Tsu-Esaki model. ${ }^{12,13}$ We follow the approach given by Gehring. ${ }^{6}$ The current going from metal to semiconductor carried by electrons that have a wave vector component in the $\mathrm{x}$-direction perpendicular to the interface between $k_{x}$ and $k_{x}+d k_{x}$ is

$$
d J_{m \rightarrow s}=q \Gamma\left(\phi_{t u n}\right) v_{x} g\left(k_{x}\right) f_{F D m}\left(1-f_{F D s}\right) d k_{x} .
$$

The velocity in the x-direction: $v_{x}=\frac{\hbar k_{x}}{m_{m}}$, where $m_{m}$ is the electron mass in the metal.

The density of states in the metal in $k$-space is $g\left(k_{x}\right)=2 \int_{0}^{\infty} \int_{0}^{\infty} \frac{1}{4 \pi^{3}} d k_{y} d k_{z}$. The factor 2 arises from spin degeneracy.

We use the notations: $\phi_{x}=\frac{\hbar^{2} k_{x}^{2}}{2 m_{m}}$ and $\varphi_{/ /}=\frac{\hbar^{2} k_{/ /}^{2}}{2 m_{m}}=\frac{\hbar^{2}}{2 m_{m}}$ $\left(k_{y}^{2}+k_{z}^{2}\right)$ for the energies in the $x$-direction and parallel to the gate metal surface, respectively.

The Fermi-Dirac distribution functions for the metal and the AlGaN semiconductor are $f_{F D m}$ and $f_{F D s}$, respectively,

$$
f_{F D m}=\frac{1}{1+\exp \left\{\frac{q\left(\phi_{x}+\phi_{/ /}-\varphi_{F}\right)}{k T}\right\}} .
$$

For the AlGaN semiconductor region, we assume that $1-f_{F D s}$ $\approx 1$.

The quantum mechanical tunneling probability through the barrier can be calculated in the case of a triangular barrier as $^{14}$

$$
\Gamma\left(\phi_{\text {tun }}\right)=\exp \left\{-\frac{\alpha_{s}}{E}\left(\varphi_{F}+\varphi_{B}-\phi_{\text {tun }}\right)^{3 / 2}\right\}
$$

where $\alpha_{s}=\frac{4 \sqrt{2 m_{s} q}}{3 \hbar}$ and $m_{s}$ is the electron mass in the semiconductor. $E$ is the electric field. The metal Fermi level is given by $\varphi_{F}$ and the barrier height, i.e., the distance between top of the barrier and the metal Fermi level, is given by $\varphi_{B}$.

Very often, a wrong expression is used for the electron energy $\phi_{\text {tun }}$ in Eq. (3). Some authors take $\phi_{\text {tun }}=\phi_{x}$, while others take $\phi_{\text {tun }}=\phi_{x}+\phi_{/ / \cdot}{ }^{1,6}$ However, it is easy to see that the electron wave function in the metal can be described by

$$
\psi_{m}=\left\{A \exp \left(i k_{x} x\right)+B \exp \left(-i k_{x} x\right)\right\} \exp \left(i k_{y} y+i k_{z} z\right) .
$$

Note that this equation contains a wave in the negative $x$ direction, which is the reflected wave at the metalsemiconductor interface. The electron wave in the semiconductor is given by

$$
\psi_{s}=C \exp \left(-\kappa_{x} x\right) \exp \left(i k_{y} y+i k_{z} z\right)
$$

where $\kappa_{x}$ described the damping of the wave in the semiconductor.

This is only valid very close to the metal interface, such that we may approximate the barrier potential by a fixed value $\varphi_{F}+\varphi_{B}$ and we can ignore the triangular nature of the barrier shape. Using the appropriate boundary conditions and Schrödinger equation, we can calculate

$$
\begin{aligned}
\kappa_{x}^{2} & =\frac{2 m_{s}}{\hbar^{2}}\left(\varphi_{F}+\varphi_{B}-\phi\right)+k_{y}^{2}+k_{z}^{2} \\
& =\frac{2 m_{s}}{\hbar^{2}}\left(\varphi_{F}+\varphi_{B}-\phi_{x}-\frac{m_{s}-m_{m}}{m_{s}} \phi_{/ /}\right),
\end{aligned}
$$

where $\phi$ is the total electron energy $\phi=\phi_{x}+\phi_{/ /}$.

Therefore, energy $\phi_{t u n}$ is

$$
\phi_{\text {tun }}=\phi_{x}+\frac{m_{s}-m_{m}}{m_{s}} \phi_{/ /}
$$

Inserting Eq. (4) into Eq. (2) and changing the integral of Eq. (1) over $k_{x}$ into an integral over $\phi_{x}$ gives

$$
\begin{aligned}
J_{m \rightarrow s}= & \left.\frac{4 \pi m_{m} q^{3}}{h^{3}} \int_{0}^{\varphi_{F}+\varphi_{B} \varphi_{F}+\varphi_{B}-\phi_{x}} \int_{0}^{3 / 2}\right\} \\
& \times \frac{\exp \left\{-\frac{\alpha_{s}}{E}\left(\varphi_{F}+\varphi_{B}-\phi_{t u n}\right)^{3 / 2}\right.}{1+\exp \left\{\frac{q\left(\phi_{x}+\phi_{/ /}-\varphi_{F}\right)}{k T}\right\}} d \phi_{/ /} d \phi_{x} .
\end{aligned}
$$

In deriving Eq. (5), we have transformed the integral over $d k_{y} d k_{z}$ using $d k_{y} d k_{z}=\frac{2 \pi m_{m}}{\hbar^{2}} d \varphi_{/ /}$.

The thermionic emission can be found from Eq. (5) by taking the integral boundaries for $\phi_{/ /}$from 0 to infinity and those for $\phi_{x}$ from $\varphi_{F}+\varphi_{B}$ to infinity and approximating the Fermi-Dirac distribution by the Boltzmann distribution. Then Eq. (5) can be calculated analytically

$$
J_{m \rightarrow s}=\frac{4 \pi m_{m} q k^{2} T^{2}}{h^{3}} \exp \left\{\frac{-q \varphi_{B}}{k T}\right\}
$$

which is the standard expression for thermionic emission. Note that we did not take barrier lowering effects, e.g., due to image charge, into account, which is allowable since the barrier height will in practice be used as a fitting parameter. $^{4}$

\section{B. Square barrier}

In case of a square barrier, i.e., a barrier with a constant potential $\varphi_{F}+\varphi_{B}$, irrespective of position $x$, the expression for the current changes by using a modified equation for Eq. (2). In this case, ${ }^{14}$ 


$$
\Gamma_{s q}\left(\phi_{\text {tun }}\right)=\exp \left\{-2 d \frac{\left[2 m_{s}\left(\varphi_{F}+\varphi_{B}-\phi_{\text {tun }}\right)\right]^{1 / 2}}{\hbar}\right\} .
$$

We will use the summation of Eqs. (5) and (6) to describe the direct current through an ideal barrier. There is also an electron flow $J_{s \rightarrow m}$ from the semiconductor to the metal. This current is, however, so low that it is only of importance at very low bias voltages that we will not consider.

\section{Patch models}

Patch models assume that the junction is for the larger part ideal and has a high barrier height $\varphi_{B}$. A barrier height in the order of $1.5-2 \mathrm{eV}$ would fit to the material parameters and the measured threshold voltage. Such a barrier would hardly conduct any current. Numerous very small defect patches carry almost all current. We can use the ideal barrier model to calculate the current if we assume that there is an effective total patch area and an effective barrier height. However, since we do not know how the potential or the electric field is shaped inside such patch, we have to consider various possibilities and compare the results of these with the measurements.

\section{Patch model 1 (the constant field patch model)}

This model assumes that the electric field in the patch is constant between the metal and the 2-DEG. See Fig. 2(a) where the potential inside the patch is displayed by the thick line. This is the simplest approximation for the field that can be made.

\section{Patch model 2 (the zero field patch model)}

This model assumes that the patches have a reduced barrier height but constant potential (zero electric field) in the patch up to a depth $d_{\text {patch }}$ where the patch potential equals the potential of the surrounding ideal barrier. See Fig. 2(b) where the potential inside the patch is displayed by the thick line. The current can be calculated by multiplying the transmission of the constant potential part of the barrier $(0<x<$ $\left.d_{\text {patch }}\right)$ with that of the triangular barrier $\left(x>d_{\text {patch }}\right)$. This can be done by using Eq. (7) and modifying Eq. (5) to

$$
J_{m \rightarrow s}=\frac{4 \pi m_{m} q^{3}}{h^{3}} \int_{0}^{\varphi_{F}+\varphi_{B}} \int_{0}^{\varphi_{F}+\varphi_{B}-\phi_{x}} \frac{\Gamma_{s q}\left(\phi_{t u n}\right) \Gamma\left(\phi_{t u n}\right)}{f_{F D m}\left(\phi_{x}\right)} d \phi_{/ /} d \phi_{x} .
$$

\section{Patch model 3 (the zero potential patch model)}

This model looks much like the zero field model with the modification that the flat potential is taken to be zero. In fact, it assumes that the patch acts as an extension of the metal up to the point $x=d_{\text {patch }}$. This gives the electrons a free ride up to a certain depth inside the barrier. This means that we can calculate the current by assuming an ideal triangular barrier with reduced barrier height but with an electric field equal to the electric field in the surrounding area.

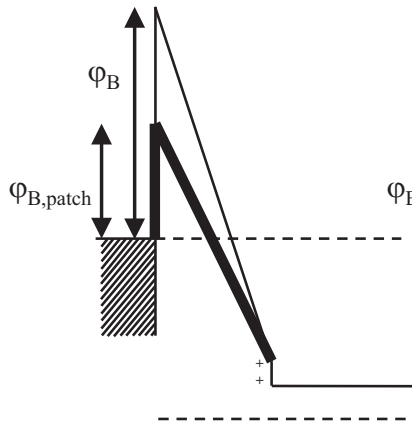

(a)

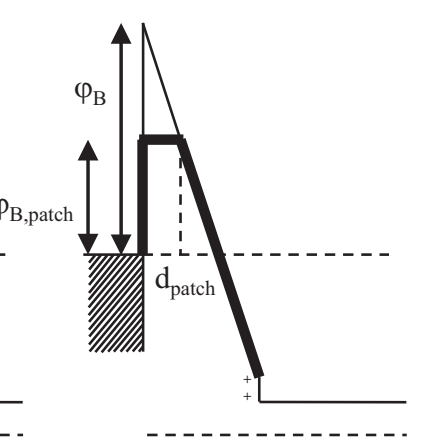

(b)
FIG. 2. (a) The constant field patch model with triangular potential barrier. (b) Zero field patch model. The potential is constant to a depth $d_{\text {patch }}$ and equal to the surrounding potential for $x>d_{\text {patch }}$. In both models, the potential inside the patch is indicated by the thick lines.

\section{TRAP ASSISTED TUNNELING}

We will first give an expression for trapping of an electron from the metal by a trap in the AlGaN semiconductor when the barrier has a square potential. This has been described by Lundström and Svensson ${ }^{15}$ in a way fully consistent with the theory in Sec. II. We will extend the theory from the square potential case to that of a triangular potential barrier. If the barrier has a square potential, the total capture probability for electrons with an energy equal to the trap energy is ${ }^{15}$

$$
\begin{aligned}
\wp= & \int_{0}^{k_{0}} p d k_{/ /}=\frac{4 \hbar m_{s}}{m_{m}^{2}} \frac{\alpha}{\alpha^{2}+k_{0}^{2}} \\
& \times \int_{0}^{k_{0}} \exp \left\{-2 \sqrt{\alpha^{2}+k_{/ /}^{2}} W\right\} \sqrt{k_{0}^{2}-k_{/ /}^{2}} k_{/ /} d k_{/ /} .
\end{aligned}
$$

In this expression, $W$ is the distance of the trap from the gate metal and $\alpha^{2}=\frac{2 m_{r} \varphi_{t}}{\hbar^{2}}$ where $\varphi_{t}$ is the trap potential measured from the bottom of the conduction band. The electron energy equals the trap potential. This assumption is justified because zero phonon transitions are dominating the capture process. ${ }^{16}$ Hence,

$$
k_{x}^{2}+k_{y}^{2}+k_{z}^{2}=k_{x}^{2}+k_{/ /}^{2}=k_{0}^{2}=\frac{2 m_{m}\left(\varphi_{F}+\varphi_{B}-\varphi_{t}\right)}{\hbar^{2}} .
$$

In the case of a triangular barrier, the trap assisted tunneling model, in its simplest form, assumes that there are traps uniformly distributed throughout the AlGaN layer that have an energy level $\varphi_{t}$ below the conduction band. Electrons from the metal may tunnel to the traps with a probability $P_{1}$ and from there tunnel to the semiconductor with a probability $P_{2}$. An illustration of this process is shown in Fig. 3.

The Schrödinger equation for the triangular barrier is

$$
\nabla^{2} \psi=\frac{2 m_{s}}{\hbar^{2}}\left\{\varphi_{F}+\varphi_{B}-E . x-\phi\right\} \psi .
$$

There is no analytical solution to the Schrödinger equation of a triangular barrier, but we can get an approximate solution by splitting the variables (for simplicity, only the $x$ - 


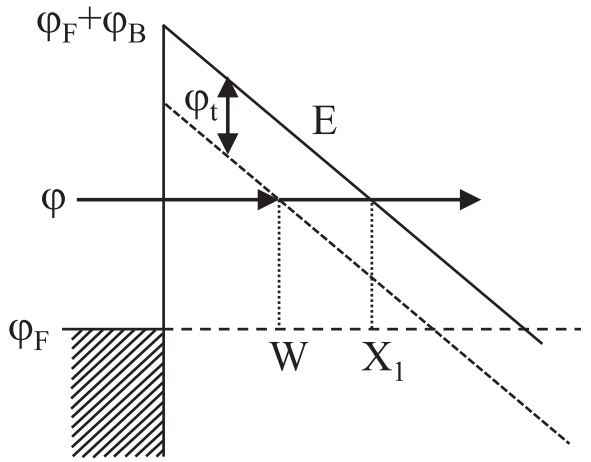

FIG. 3. Tunneling by electrons having energy $\phi$ assisted by traps at energy level $\varphi_{t}$ below the conduction band in AlGaN .

and $y$-components are taken into account. It is straightforward to extend the final result also to the $z$-direction)

$$
\begin{gathered}
\psi(x, y)=\xi(x) \chi(y), \\
\frac{1}{\xi} \frac{\partial^{2} \xi}{\partial x^{2}}+\frac{1}{\chi} \frac{\partial^{2} \chi}{\partial y^{2}}=\frac{2 m_{s}}{\hbar^{2}}\left\{\varphi_{F}+\varphi_{B}-E \cdot x-\phi\right\} .
\end{gathered}
$$

We now assume that $\chi=\exp \left(i k_{y} y\right)$, so the Schrödinger equation reduces to:

$$
\begin{aligned}
\frac{1}{\xi} \frac{\partial^{2} \xi}{\partial x^{2}} & =\frac{2 m_{s}}{\hbar^{2}}\left\{\varphi_{F}+\varphi_{B}-E \cdot x-\phi\right\}+k_{y}^{2} \\
& =\frac{2 m_{s}}{\hbar^{2}}\left\{\varphi_{F}+\varphi_{B}-\phi_{\mathrm{tun}}-E \cdot x\right\} .
\end{aligned}
$$

This is an equation of the shape: $\frac{1}{\xi} \frac{\partial^{2} \xi}{\partial x^{2}}=A-B x$ with $A=$ $\frac{2 m_{s}\left(\varphi_{F}+\varphi_{B}-\phi_{\text {tun }}\right)}{\hbar^{2}}$ and $B=\frac{2 m_{s} E}{\hbar^{2}}$.

Although there is no general analytical solution, we can try a function like

$$
\xi=\exp \left\{-\int_{0}^{x}\left(A-B x^{\prime}\right) d x^{\prime}\right\} .
$$

Then,

$$
\frac{1}{\xi} \frac{\partial^{2} \xi}{\partial x^{2}}=A-B x+\frac{B}{2 \sqrt{A-B x}}
$$

This is a Schrödinger equation of a barrier that is deviating from a triangular shape by the last term on the right hand side. If we normalize Eq. (11), we get

$$
\begin{aligned}
\frac{1}{A-B x} \frac{1}{\xi} \frac{\partial^{2} \xi}{\partial x^{2}} & =1+\frac{B}{2(A-B x)^{1.5}} \\
& =1+\frac{E}{2 \sqrt{\frac{2 m_{s}}{\hbar^{2}}}\left\{\varphi_{F}+\varphi_{B}-\phi_{t u n}-E . x\right\}^{1.5}} \\
& =g(x, \text { par }) .
\end{aligned}
$$

The right hand side deviates most from 1 when $\varphi_{F}+\varphi_{B}$ - $\phi_{\text {tun }}$ is minimal and $E x$ is high. From Fig. 3, it is clear that $E x=\varphi_{F}+\varphi_{B}-\varphi_{t}-\phi \leq \varphi_{F}+\varphi_{B}-\varphi_{t}-\phi_{\text {tun }} \Rightarrow \varphi_{F}+$
$\varphi_{B}-\phi_{\text {tun }}-E x \geq \varphi_{t}$. So the right hand side of Eq. (12) is always finite and the largest deviation from a triangular barrier occurs for low values of $\varphi_{t}$. Let us assume that $\varphi_{t}$ $\geq 0.1 \mathrm{eV}$. The electric field is at maximum $3 \mathrm{~V}$ over $20 \mathrm{~nm}$ before complete depletion of the 2-DEG takes place and the vertical field cannot be further increased, so $E_{\text {max }}$ $\approx 1.5 \times 10^{8} \mathrm{~V} / \mathrm{m}$. In Fig. 4, we have plotted the right hand side of Eq. (12) $g(x$, par $)$ as function of depth for several values of par $=\varphi_{F}+\varphi_{B}-\phi_{\text {tun }}: 0.1,0.2,0.5,1.0$, and $3.0 \mathrm{eV}$, with $E=E_{\text {max }}$. We can make the following observations.

The curves tend to infinity when $E_{\max } x$ approaches $\varphi_{F}$ $+\varphi_{B}-\phi_{\text {tun }}$. As said, this will never occur. The curve for the case of $0.1 \mathrm{eV}$ rises already considerably above 1 for low values of $x$, so in this case Eq. (10) is not a good wave function to use.

For values of $\varphi_{F}+\varphi_{B}-\phi_{\text {tun }} \geq 0.2 \mathrm{eV}$, the deviation of Eq. (12) from unity at low values of $x$ is minimal. Therefore, we can conclude that Eq. (10) is a good approximation for the wave function for traps deeper than $0.2 \mathrm{eV}$ below the conduction band. This solution can also be used for slightly shallower traps without significant error, especially at lower values of $E$.

This leads to a modified version of Eq. (8) for the triangular barrier

$$
\begin{aligned}
\wp_{1}= & \frac{4 \hbar m_{m}}{m_{s}^{2}} \frac{\alpha}{\alpha^{2}+k_{0}^{2}} \\
& \times \int_{0}^{k_{0}} \exp \left\{-2 \int_{0}^{W} \sqrt{\frac{2 m_{s}}{\hbar^{2}}\left\{\varphi_{F}+\varphi_{B}-\phi_{\text {tun }}-E x\right\}} d x\right\} k_{x} k_{/ /} d k_{/ /} .
\end{aligned}
$$

If we replace $k_{x}$ by $k_{x}=\sqrt{k_{0}^{2}-k_{/ /}^{2}}$ and $\phi_{\text {tun }}$ by $\phi_{\text {tun }}=\phi-\frac{m_{m}}{m_{s}} \phi_{/ /}=\phi-\frac{\hbar^{2} k_{/ /}^{2}}{2 m_{s}}$, we can integrate Eq. (13) over $k_{/ /}$and calculate $\wp_{1}$.

An equation for the de-trapping probability can be derived analogous to the derivation of $\wp_{1}$. Referring to Fig. 3 at reverse bias voltage $V_{r}$, the electron Fermi level in the semiconductor is $V_{r}$ below $\varphi_{F}$. We can calculate the probability $\wp_{2}$ of a trap located at $W$ for capturing an electron from

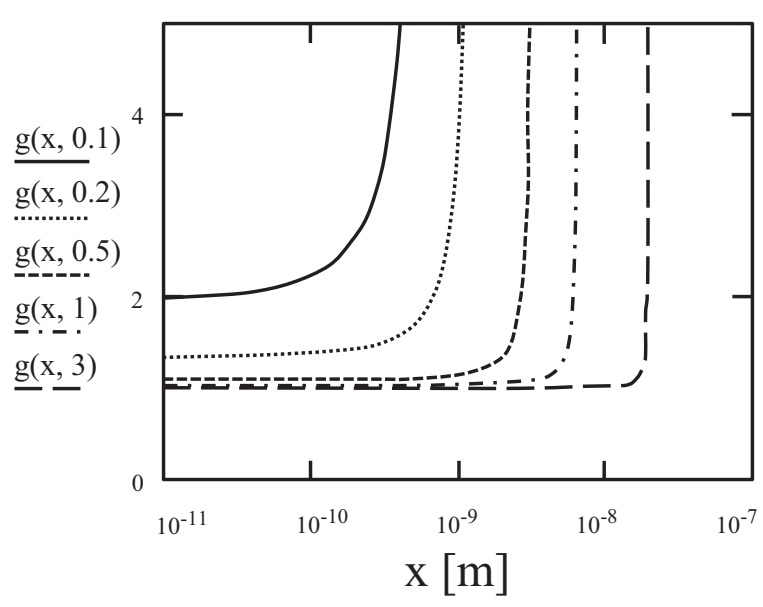

FIG. 4. Deviation of triangular barrier as function of depth for different trap energy levels $\varphi_{t}$. 
the semiconductor that has the right energy, i.e., an energy $\varphi_{B}-\varphi_{t}-E W+V_{r}$ above the conduction band minimum in the semiconductor. Using detailed balance considerations, this probability equals the de-trapping probability of an electron from the trap into the semiconductor. Assuming that the conduction band minimum is very close to the Fermi level, this means

$$
k_{0}^{2}=\frac{2 m_{s}\left(\varphi_{B}-\varphi_{t}-E W+V_{r}\right)}{\hbar^{2}} .
$$

We can calculate $\wp_{2}$ simply by using Eq. (13), taking $\varphi_{t}$ as barrier height and replacing $W$ by $\varphi_{t} / E$. This gives

$$
\begin{aligned}
\wp_{2}= & \int_{0}^{k_{0}} p d k_{/ /}=\frac{4 \hbar}{m_{s}} \frac{\alpha}{\alpha^{2}+k_{0}^{2}} \\
& \times \int_{0}^{k_{0}} \exp \left\{-2 \int_{0}^{\varphi_{t} / E} \sqrt{\frac{2 m_{s}}{\hbar^{2}}\left\{\varphi_{t}-E x\right\}+k_{/ /}^{2}} d x\right\} k_{x} k_{/ /} d k_{/ /}
\end{aligned}
$$

The term with $k_{/ /}$in the exponent arises because there are multiple orbits available for de-trapping into the semiconductor with different $k_{/ /}$levels. The argument is completely analog to the reverse case in which free electrons from the semiconductor are trapped into the barrier.

The next step is to calculate the gate current from $\wp_{1}$ and $\wp_{2}$. Suppose at a certain depth $W$, there is a trap concentration $N_{t}$. Suppose further that a fraction $n$ of these traps are occupied by electrons and therefore do not trap more electrons. The concentration of empty traps that are capable of capturing electrons is $N_{t}-n$. The capture rate of the traps is given by $f_{F D m} \wp_{1}\left(N_{t}-n\right)$, while the de-trapping rate is $\wp_{2} n$. In equilibrium, these rates must be equal, so

$$
\frac{\wp_{2}}{f_{F D m} \wp_{1}}=\frac{N_{t}-n}{n} \text {. }
$$

The capture rate therefore is: $\quad R=\frac{N_{t} f_{F D m} \wp_{1} \wp_{2}}{f_{F D m} \wp_{1}+\wp_{2}}=N_{t}$ $\left(\frac{1}{f_{F D m} \wp_{1}}+\frac{1}{\wp_{2}}\right)^{-1}$.

The current contribution of these traps is $\Delta J_{T A T}=q R$. The total TAT current is the integral of $\Delta J_{\text {TAT }}$ over all traps, so over all values of $W$ running from zero for energies equal to $\varphi_{F}+\varphi_{B}-\varphi_{t}$ to very large values for very low energies. In practice, energies below the Fermi level will hardly contribute anymore. So we can write for the total TAT current

$$
J_{T A T}=q N_{t} \int_{0}^{\infty}\left(\frac{1}{f_{F D m} \wp_{1}}+\frac{1}{\wp_{2}}\right)^{-1} d W .
$$

The only variables depending on $W$ in this expression are $\wp_{1}$ and $f_{F D m}$. Integration has to be done numerically after substituting Eqs. (13) and (14) into Eq. (15).

The expression (15) describes the current contribution caused by one trap level with an energy $\varphi_{t}$ below the conduction band that is uniformly distributed throughout the semiconductor barrier. If there are several trap levels available, each of them will contribute according to Eq. (15) and all contributions have to be summed.

\section{MEASUREMENTS}

Measurements were done of the gate to source current in a Schottky diode with a square gate area minimizing the influence of the gate edge. Voltages are applied between the negative threshold voltage and zero volt. In that case, the electric field at the gate edge is low because the 2-DEG is only (partly) depleted underneath the gate and not next to the gate. The current flow as well as the electric field can essentially be treated as one-dimensional and be compared to the models developed above. The diodes were processed by United Monolithic Semiconductors (UMS: www.ums-gaas.com) in Germany. ${ }^{17}$ The process technology has been described before, including TEM pictures of the layer structure. ${ }^{18}$ The HEMT structure is grown on SI-SiC substrate by MOVPE. Device isolation was done by ion implantation. The ohmic contact was made by evaporating $\mathrm{Ti} / \mathrm{Al} / \mathrm{Ni} / \mathrm{Au}$, which was annealed at $870^{\circ} \mathrm{C}$ by RTA. A SiN layer was deposited on the $\mathrm{AlGaN}$ and a gate opening was etched in the $\mathrm{SiN}$ layer using a $\mathrm{CF}_{4}$ based ICP process. After that a NiPtAu layer was deposited as Schottky contact and annealed at $400{ }^{\circ} \mathrm{C}$. The resulting sheet resistance of the 2-DEG was $550 \Omega$ / square. We used the following material parameter settings: $\varepsilon_{r}$ $=9.5$ for the AlGaN, $m_{m}=0.1 m_{0}, m_{s}=0.23 m_{0}$ with $m_{0}$ the vacuum electron mass. ${ }^{19}$ Since the calculated results are not very sensitive to the metal Fermi level, we take $\varphi_{F}=5 \mathrm{eV}$ more or less arbitrarily, which is a suitable value for most gate metals. The parameters describing the diode structure are $d_{\text {eff }}=25 \mathrm{~nm}$ as effective barrier thickness, $A=10^{-8} \mathrm{~m}^{2}$ as gate area, and $\Delta V=0.4 \mathrm{~V}$ as the sum of the conduction band offset between $\mathrm{AlGaN}$ and GaN and the GaN flatband voltage, see Fig. 5. The factor $\Delta V$ contributes to the total electric field across the barrier. From Fig. 5, the electric field in the barrier is

$$
E=\frac{V_{r}+\varphi_{B}-\varphi_{\text {flatband }}-\Delta \varphi_{C}}{d_{\text {eff }}} \text { and } \Delta V=\varphi_{\text {flatband }}+\Delta \varphi_{C} .
$$

In practice, $\Delta V$ is used as a fitting parameter that shifts the $I-V$ characteristics along the voltage axis.

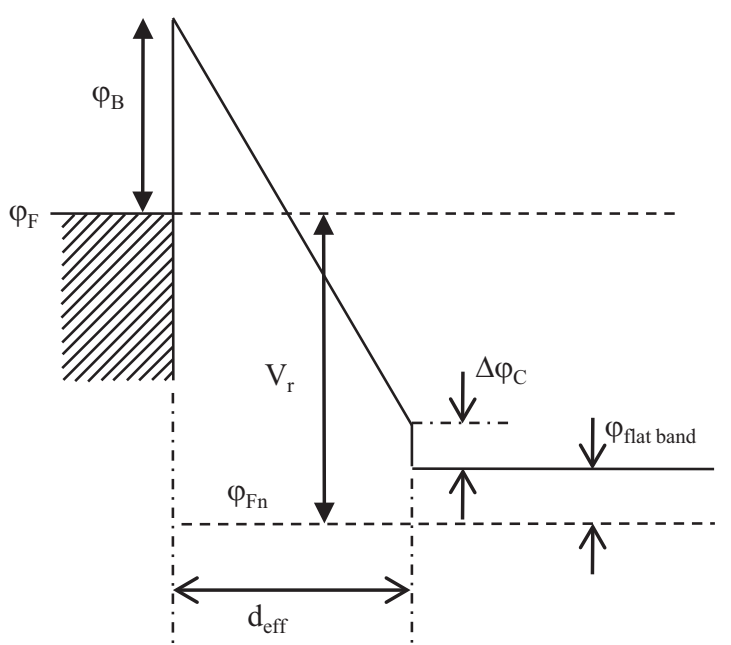

FIG. 5. The influence of the flatband voltage $\varphi_{\text {flat band }}$ and conduction band offset $\Delta \varphi_{C}$ between $\mathrm{AlGaN}$ and $\mathrm{GaN}$ on the total electric field in the barrier. $\varphi_{f n}$ is the electron Fermi level in the semiconductor. 
A comparison is made between the various theoretical models and the measured data in the following sections.

\section{A. The trap assisted tunneling model}

This model assumes that each type of trap is distributed uniformly throughout the $\mathrm{AlGaN}$ region and can be characterized by only two parameters: the concentration $N_{t}$ and the energy level $\varphi_{t}$. Each trap type will contribute a current $J_{T A T}$ according to Eq. (15). Every trap contribution is added to the direct current through the barrier given by the sum of Eqs. (5) and (6). Having many traps and their contributions to the total current raises the possibility that the model can become Ptolemaeic, i.e., that we introduce so many traps and associated fitting parameters, that we can fit any data without obtaining a model that makes physically sense. Therefore, we limit the number of traps in the model to two, which gives us four extra fitting parameters, next to the ones we already have for the direct current. Fig. 6 shows the measured and calculated data. Note that the threshold voltage of the device is $-2.5 \mathrm{~V}$. At lower voltages, the vertical field underneath the gate cannot increase any further because the 2-DEG is completely depleted. The measured data therefore become independent of voltage below the threshold voltage and the theories developed in this paper lose their validity. At higher temperatures, the direct current dominates. This allows us to accurately extract the barrier height from the data at $175^{\circ} \mathrm{C}$. This results in $\varphi_{B}=0.97 \mathrm{eV}$. The trap parameters are optimized to give the best fit to the data at $25^{\circ} \mathrm{C}$. One trap is at an energy level $\varphi_{t l}=0.58 \mathrm{eV}$ and at a concentration of $N_{t 1}=6 \times 10^{14} \mathrm{~cm}^{-3}$ and the other trap has $\varphi_{t 2}$ $=0.35 \mathrm{eV}$ at a concentration of $N_{t 2}=1.5 \times 10^{15} \mathrm{~cm}^{-3}$.

As can be seen in Fig. 6, the traps add very distinct humps in the current curves, especially at lower temperatures where TAT dominates the total current. However, the measured data at $25^{\circ} \mathrm{C}$ do not show these typical trap signatures, suggesting that TAT does not occur in our samples. Of course, it is possible to add enough trap types in the theory, each with its own energy level and concentration, to obtain also a smooth $I-V$ curve at $25^{\circ} \mathrm{C}$, but it is very unlikely that such a model would represent reality.

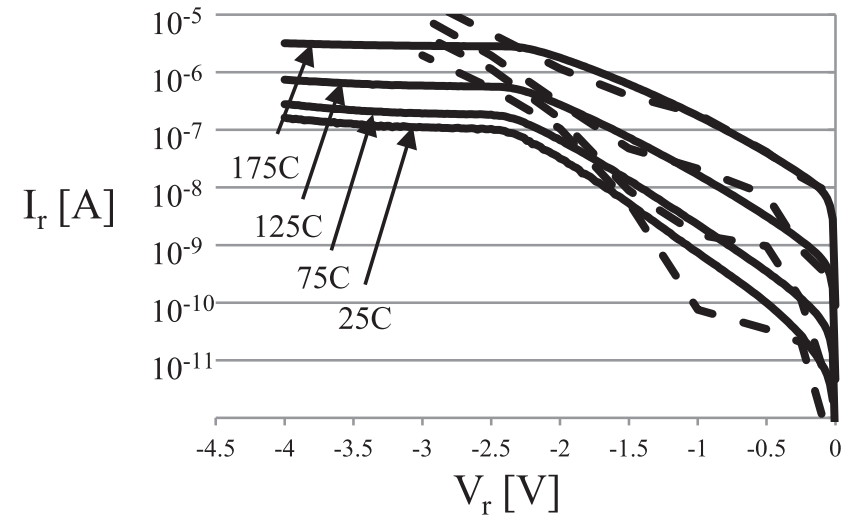

FIG. 6. Measured current-voltage data (solid lines) at 25, 75, 125, and $175^{\circ} \mathrm{C}$ and calculations (striped lines) using a TAT model with two distinct traps at 0.35 and $0.58 \mathrm{eV}$ and a barrier height of $0.97 \mathrm{eV}$.

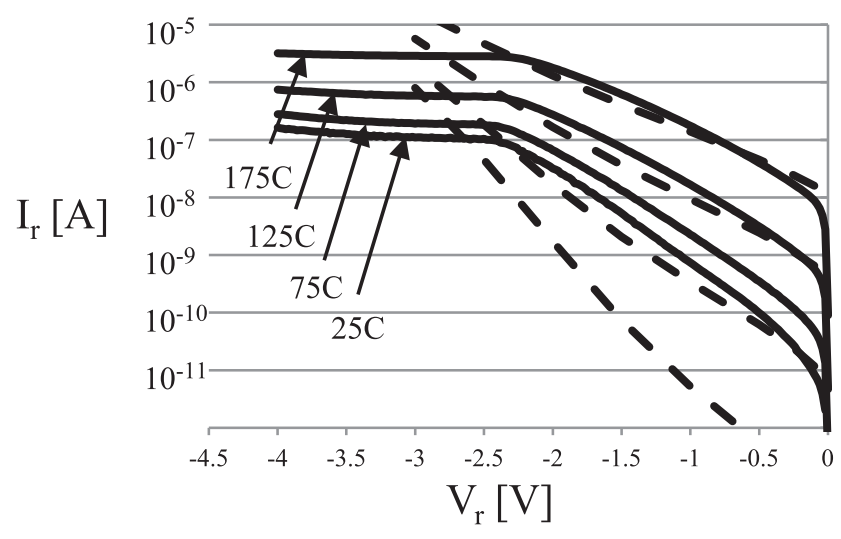

FIG. 7. Comparison between measurements and calculations for the case of an ideal barrier with a height of $0.95 \mathrm{eV}$.

\section{B. The ideal barrier}

In this model, we only use the direct current through the defect-free barrier given by the sum of Eqs. (5) and (6). We treat the barrier height $\varphi_{B}$ as a fitting parameter that we fit to the high temperature measurements at $175^{\circ} \mathrm{C}$, which results in a barrier height value of $0.95 \mathrm{eV}$. The comparison between measured and calculated values is shown in Fig. 7.

From Fig. 7, we conclude that, although the high temperature data can be reproduced, the model clearly fails at the lower temperatures. Therefore, we will evaluate the patch models as described in Sec. II.

\section{The constant field patch model}

In Fig. 8, the calculations are compared with the measured data for a patch area that is $2 \times 10^{-4}$ times the total area with an effective patch barrier height of $0.58 \mathrm{eV}$ (see Sec. II $\mathrm{C} 1$ ). Note that the calculated current includes the thermionic emission current. This gives a good fit with the measured data, indicating that indeed this patch model may explain the data.

\section{The zero field patch model}

Calculations show that if $\varphi_{B, \text { patch }}$ is low, the current is dominated by thermionic emission and does not depend on voltage (see Sec. IIC2). If, on the other hand, $\varphi_{B, p a t c h}$ is taken high enough such that thermionic emission does not

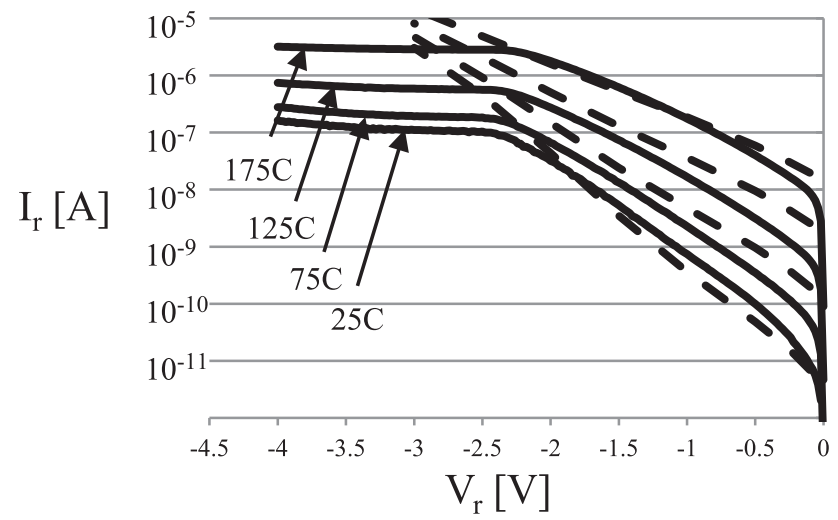

FIG. 8. Measured data and calculations using a defect patch model with triangular barrier, i.e., constant electric field throughout the patch. 


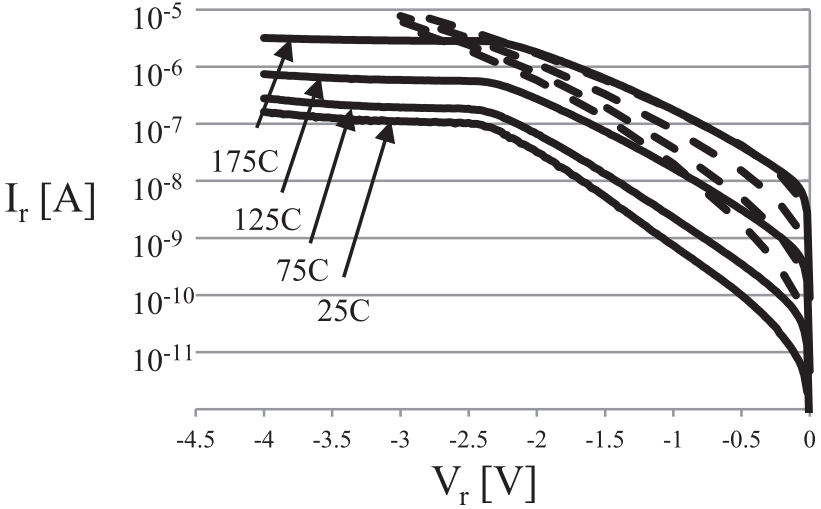

FIG. 9. Measured data and calculations using zero potential defect patch model.

dominate, the total current is far too low to account for the observations. Therefore, the zero field patch model cannot explain the data.

\section{E. The zero potential patch model}

We can calculate the current by assuming an ideal triangular barrier with reduced barrier height and reduced thickness but with an electric field equal to the electric field in the surrounding area (see Sec. II C 3). We can obtain a perfect fit at the highest temperature by setting the patch area to the total area ratio at $10^{-5}$ and the patch barrier height to $0.60 \mathrm{eV}$. The calculations, however, deviate dramatically from the data at lower temperature (see Fig. 9). It is clear that the zero potential patch model also cannot explain the measured data.

\section{CONCLUSIONS}

We have developed theoretical models for currents in triangular and square Schottky barriers caused by tunneling, thermionic field emission, thermionic emission, and trap assisted tunneling. The models are compared with measurements on a large area Schottky diode on an AlGaN-GaN structure as used in high electron mobility transistors. Comparison was done over a voltage range between the negative threshold voltage and zero volt and over a temperature range from $25^{\circ} \mathrm{C}$ to $175^{\circ} \mathrm{C}$. The measured data can be understood best by assuming that the current flows through only a very small fraction of the diode area, possibly through defect patches caused by epitaxial growth or gate processing. These defect patches have a low Schottky barrier height. Best agreement with measurements was obtained assuming that the defect area is only a fraction of $2 \times 10^{-4}$ of the total diode area and the defect barrier height is $0.58 \mathrm{eV}$ in the samples. A constant electric field in the defect patches is assumed to obtain the best fit with the data. Future research is needed to identify the nature and cause of the defects in order to reduce the Schottky current.

\section{ACKNOWLEDGMENTS}

The author likes to thank United Monolithic Semiconductors (UMS), Ulm, Germany for providing the Schottky diodes and Fredrik Allerstam at NXP Semiconductors, Nijmegen, The Netherlands for the measurements and helpful discussions. This work was supported by the GHz Centre at Chalmers University, Gothenburg, Sweden.

${ }^{1}$ S. Karmalkar and D. M. Sathaiya, "Unified closed-form model of thermionic-field and field emissions through a triangular potential barrier," Appl. Phys. Lett. 82(9), 1431-1433 (2003).

${ }^{2}$ M. P. Huong, Y. H. Wang, and W. J. Chang, "Current transport mechanism in trapped oxides: A general trap-assisted tunneling model," J. Appl. Phys. 86(3), 1488-1491 (1999).

${ }^{3}$ S. Karmalkar, D. M. Sathaiya, and M. S. Shur, "Mechanism of the reverse gate leakage in AlGaN/GaN high electron mobility transistors," Appl. Phys. Lett. 82(22), 3976-3978 (2003).

${ }^{4}$ F. Jimenez-Molinos, F. Gamiz, A. Palma, P. Cartujo, and J.A. Lopez-Villanueva, "Direct and trap-assisted elastic tunneling through ultrathin gate oxides,” J. Appl. Phys. 91(8), 5116-5124 (2002).

${ }^{5}$ S. Fleischer, P. T. Lai, and Y. C. Cheng, "Simplified closed-form trapassisted tunneling model applied to nitride oxide dielectric capacitors," J. Appl. Phys. 72(12), 5711-5715 (1992).

${ }^{6}$ A. Gehring, "Simulation of tunneling in semiconductor devices," Ph.D. dissertation (Technical University Vienna, 2003).

${ }^{7}$ X. R. Cheng, Y. C. Cheng, and B. Y. Liu, "Nitridation-enhanced conductivity behavior and current transport mechanism in thin thermally nitride $\mathrm{SiO}_{2}$," J. Appl. Phys. 63(3), 797-902 (1988).

${ }^{8}$ T. Sawada, N. Kimura, K. Imai, K. Suzuki, and K. Tanahashi, "Interpretation of current transport properties at Ni/n-GaN Schottky interfaces,” J. Vac. Sci. Technol. B 22(4), 2051-2058 (2004).

${ }^{9}$ J. Spradlin, S. Dogan, J. Xie, R. Molnar, A. A. Baski, and H. Morkoç, "Investigation of forward and reverse current conduction in GaN films by conductive atomic force microscopy," Appl. Phys. Lett. 84(21), 41504152 (2004).

${ }^{10}$ S.-H. Park, H. Kim, K.-M. Song, and D.-W. Kim, "Observations of barrier inhomogeneity in Pt/ $\alpha$-plane n-type GaN Schottky contacts," J. Korean Phys. Soc. 58(5), 1356-1360 (2011).

${ }^{11}$ F. Giannazzo, F. Roccaforte, F. Iucolano, V. Raineri, F. Ruffino, and M. G. Grimaldi, "Nanoscale current transport through Schottky contacts on wide bandgap semiconductors," J. Vac. Sci. Technol. B 27(2), 789-794 (2009).

${ }^{12}$ C. B. Duke, Tunneling in Solids (Academic, 1969).

${ }^{13}$ R. Tsu and L. Esaki, "Tunneling in a finite superlattice," Appl. Phys. Lett. 22(11), 562-564 (1973).

${ }^{14}$ S. Gasiorowicz, Quantum Physics (John Wiley and Sons, New York, 1974).

${ }^{15}$ I. Lundström and C. Svensson, "Tunneling to traps in insulators," J. Appl. Phys. 43(12), 5045-5047 (1972).

${ }^{16}$ F. Jimenez-Molinos, A. Palma, F. Gamiz, J. Banqueri, and J. A. LopezVillanueva, "Physical model for trap-assisted inelastic tunneling in metaloxide semiconductor structures," J. Appl. Phys. 90(7), 3396-3404 (2001).

${ }^{17}$ H. Blanck, J. R. Thorpe, R. Behtash, J. Splettstößer, P. Brückner, S. Heckmann, H. Jung, K. Riepe, F. Bourgeois, M. Hosch, D. Köhn, H. Stieglauer, D. Floriot, B. Lambert, L. Favede, Z. Ouarch, and M. Camiade, "Industrial GaN FET technology," Int. J. Microwave Wireless Technol. 2, 21-32 (2010); United Monolithic Semiconductors, Orsay, France, www.umsgaas.com.

${ }^{18}$ H. Jung, R. Behtash, J. R. Thorpe, K. Riepe, F. Bourgeois, H. Blanck, A. Chuvilin, and U. Kaiser, "Reliability behavior of GaN HEMTs related to Au diffusion at the Schottky interface," Phys. Status Solidi C 6(S2), 976979 (2009).

${ }^{19}$ V. Bougrov, M. Levinshtein, S. Rumyantsev, and A. Zubrilov, Properties of Advanced Semiconductor Materials, edited by M. E. Levinshtein, S. L. Rumyantsev, and M. S. Shur (John Wiley and Sons, New York, 2001). 